\title{
FOURIER TRANSFORMS WITH ONLY REAL ZEROS ${ }^{1}$
}

\author{
CHARLES M. NEWMAN ${ }^{2}$
}

\begin{abstract}
The class of even, nonnegative, finite measures $\rho$ on the real line such that for any $b>0$ the Fourier transform of $\exp \left(-b t^{2}\right) d \rho(t)$ has only real zeros is completely determined. This result is then applied to the Riemann hypothesis.
\end{abstract}

1. Main results. The problem of determining whether a Fourier transform has only real zeros arises in two rather disparate areas of mathematics: number theory and mathematical physics. In number theory, the problem is intimately associated with the Riemann hypothesis [T, Chapter 10], while in mathematical physics it is closely connected with the Lee-Yang theorem of statistical mechanics and quantum field theory [SG], [N1], [N3]; see Kac's remarks in [P, pp. 424-426] for a discussion of the historical connection between these two topics. The results of this paper developed out of the study of certain quantum field theoretic problems, but for pedagogical reasons, we present them in the context of the Riemann hypothesis.

Following standard practice, we define the Riemann xi function as

$$
\Xi(z)=s(s-1) \pi^{-s / 2} \Gamma(s / 2) \zeta(s) / 2 ; \quad s=i z+\frac{1}{2},
$$

where $\zeta(s)$ is the Riemann zeta function. $\Xi$ is the Fourier transform of the strictly positive, even function,

$$
F(t)=\sum_{n=1}^{\infty}\left(4 n^{4} \pi^{2} e^{9 t / 2}-6 n^{2} \pi e^{5 t / 2}\right) \exp \left(-n^{2} \pi e^{2 t}\right),
$$

which satisfies

$$
F(t)=O\left(\exp \left(9|t| / 2-\pi e^{2|t|}\right)\right) \text { as }|t| \rightarrow \infty,
$$

and the Riemann hypothesis is identical to the conjecture that the zeros of $\Xi$ are all real. For a discussion of these and other facts related to the Riemann hypothesis, see [T, Chapters 1, 2, 10].

We generalize the xi function by defining for arbitrary real $b$,

$$
\Xi_{b}(z)=\int_{-\infty}^{\infty} \exp \left(i z t-b t^{2}\right) F(t) d t
$$

Received by the editors January 5, 1976 and, in revised form, May 3, 1976.

AMS (MOS) subject classifications (1970). Primary 30A08, 10H05.

${ }^{1}$ Research partially supported by National Science Foundation Grant MPS 74-04870 A01.

${ }^{2}$ NATO postdoctoral fellow on leave from Department of Mathematics, Indiana University, Bloomington, Indiana. 
so that $\Xi$ and $\Xi_{0}$ are identical. The following theorem, which is due to de Bruijn [B, Theorem 13] and is an extension of various results of Pólya, indicates that definition (1.4) is a natural one in that whenever the zeros of $\Xi_{b^{\prime}}$ are all real, then so are the zeros of $\Xi_{b}$ for $b<b^{\prime}$. This theorem of de Bruijn, when combined with the fact that all the zeros of $\Xi_{0}$ lie in the critical strip $|\operatorname{Im} z| \leqslant \frac{1}{2}$, also implies that (at least) for $b \leqslant-\frac{1}{8}$, the zeros of $\Xi_{b}$ are all real. The main number theoretic conclusion of this paper (Theorem 3 below) is the complementary fact that for some $b_{1}$, the zeros of $\Xi_{b}$ are not all real for $b>b_{1}$.

THEOREM 1. Suppose $f$ is a real, even, integrable function on $\mathbf{R}$ with $|f(t)|=$ $O\left(\exp \left(-|t|^{\gamma}\right)\right)$ as $|t| \rightarrow+\infty$ for some $\gamma>2$, such that all the zeros of the Fourier transform of $f$ lie in the strip $|\operatorname{Im} z| \leqslant \Delta$; then for $\delta \geqslant 0$, all the zeros of the Fourier transform of $\exp \left(\delta t^{2}\right) f(t)$ lie in the strip

$$
|\operatorname{Im} z| \leqslant\left[\max \left(\Delta^{2}-2 \delta, 0\right)\right]^{1 / 2}
$$

Motivated by Theorem 1 and the discussion preceding it, we define $\$ ?$ to be the class of even, nonnegative, finite measures $\rho$ on the real line such that for any $b>0$, the Fourier transform of $\exp \left(-b t^{2}\right) d \rho(t)$ has only real zeros. One group of measures belonging to $\mathcal{P}$, which was discovered by Pólya (see [B, p. 197]), consists of absolutely continuous ones with density

$$
d \rho / d t=K t^{2 m} \exp \left(-\alpha t^{4}-\beta t^{2}\right) \prod_{j}\left(1+t^{2} / a_{j}^{2}\right)
$$

where $K>0, m=0,1,2, \ldots, a_{j}>0, \Sigma\left(1 / a_{j}^{2}\right)<\infty, \alpha>0$ and $\beta$ is real (or else $\alpha=0$ and $\beta>0$ ); here and elsewhere in this paper, $t^{2 m}$ with $m=0$ means the function identically equal to 1 . While working on the Riemann hypothesis, de Bruijn discovered [B, Theorem 28] that the product of two densities of measures in $\mathcal{P}$ is again the density of a measure in $\mathscr{P}$ but could not find any measures in $\mathcal{P}$ essentially different from those of (1.5). The class $\mathcal{P}$ is also a natural one in quantum field theory where one is particularly interested in functions $V(t)$ such that $\exp (-\lambda V(t)) d t \in \mathscr{P}$ for all $\lambda>0$, and the example $V(t)=\alpha t^{4}+\beta t^{2}$ of (1.5) was in fact rediscovered by statistical mechanical methods in [SG]; Theorem 2 below shows that no other $V(t)$ is possible, thus disproving a conjecture of [N3] that $V(t)=\cosh t$ is allowed.

The next theorem gives a complete classification of $\mathcal{P}$ and shows that (1.5) yields essentially the whole class; we change from Fourier to Laplace transforms in the statement of the theorem for purposes of the proof, which is presented in $\$ 2$ of the paper.

THEOREM 2. Suppose $\rho$ is an even, nonnegative, finite measure on the real line and $Z_{b}$ is defined (for $b>0$ ) as

$$
Z_{b}(z)=\int_{-\infty}^{\infty} \exp \left(z t-b t^{2}\right) d \rho(t)
$$

then $Z_{b}$ has only pure imaginary zeros for every $b>0$ if and only if either 


$$
\rho(t)=K\left(\delta\left(t-t_{0}\right)+\delta\left(t+t_{0}\right)\right)
$$

for some $K>0$ and $t_{0} \geqslant 0$ (where $\delta\left(t-t_{0}\right.$ ) denotes the point measure of unit mass concentrated at the point $\left.t_{0}\right)$, or else $\rho$ is absolutely continuous with density

$$
\frac{d \rho}{d t}=K t^{2 m} \exp \left(-\alpha t^{4}-\beta t^{2}\right) \prod_{j}\left[\left(1+\frac{t^{2}}{a_{j}^{2}}\right) \exp \left(\frac{-t^{2}}{a_{j}^{2}}\right)\right]
$$

where $K>0, m=0,1,2, \ldots, a_{j}>0, \Sigma\left(1 / a_{j}^{4}\right)<\infty, \alpha>0$ and $\beta$ is real (or else $\alpha=0$ and $\left.\beta+\Sigma\left(1 / a_{j}\right)^{2}>0\right)$. The product in (1.8) is over a set of $j$ 's which may be empty, finite, or infinite and the condition $\beta+\Sigma\left(1 / a_{j}\right)^{2}>0$ is considered to be satisfied if $\Sigma\left(1 / a_{j}\right)^{2}=+\infty$.

REMARK 1. If we weaken the assumptions on $\rho$ to allow noneven real-valued measures, the theorem remains true provided we also allow $d \rho(t)$ in the conclusion to be $\pm t^{n} d \rho_{0}(t)$ (with $n=0$ or 1) with $\rho_{0}$ as given by (1.7) or (1.8) or else proportional to a Gaussian measure of mean $t_{0}$ and variance $\sigma^{2}$ with $t_{0}$ real and $\sigma^{2} \geqslant 0$. We do not include this result in Theorem 2 simply because the extra complications needed for the proof do not seem justified by the extra generality obtained.

THEOREM 3. There exists a real number $b_{0}$ with $-\frac{1}{8} \leqslant b_{0}<\infty$ such that $\Xi_{b}(z)$ has only real zeros when $b \leqslant b_{0}$ but has nonreal zeros when $b>b_{0}$.

Proof. This result follows immediately from Theorems 1 and 2 (see the discussion preceding Theorem 1) once we show that $F(t)$, as given by (1.2), is not of the form of (1.8). To see this, we note that one consequence of (1.8) is that

$$
d \rho / d t \geqslant K t^{2 m} \exp \left(-\left[\alpha+\sum\left(1 /\left(2 a_{j}^{4}\right)\right)\right] t^{4}-\beta t^{2}\right)
$$

while $F$ satisfies (1.3); (1.9) follows from (1.8) after application of the inequality

$$
\left(1+x^{2}\right) \exp \left(-x^{2}\right) \geqslant \exp \left(-x^{4} / 2\right) ; \quad x \in \mathbf{R},
$$

which itself may be obtained by exponentiating the elementary inequality, $\ln (1+y) \geqslant y-y^{2} / 2$ for $y \geqslant 0$.

REMARK 2. The Riemann hypothesis is the statement that $b_{0} \geqslant 0$; we make the complementary conjecture that $b_{0} \leqslant 0$. This new conjecture is a quantitative version of the dictum that the Riemann hypothesis, if true, is only barely so.

\section{Proof of Theorem 2.}

Proof of Sufficiency. The measure of (1.7) clearly belongs to 99 while the measures of (1.8) can be seen to belong to $\mathcal{P}$ since they are limits of Pólya's measures of (1.5) (via truncation of the product in (1.8)). For the sake of completeness, we note that the measures of (1.5) can themselves be obtained as limits of measures with density $d \rho / d t=Q(t) \exp \left(-c t^{2}\right)$, where $Q$ is an 
even polynomial with only pure imaginary zeros and $c>0$, since

$$
\left(1+(2 \alpha / n)^{1 / 2} t^{2}\right)^{n} \exp \left(-(2 \alpha n)^{1 / 2} t^{2}\right) \rightarrow \exp \left(-\alpha t^{4}\right)
$$

and that measures with such densities belong to $\$$ since their Laplace transforms may be expressed as a limit of polynomials of the form $Q(d / d z) P(z)$ (where $P$ is an even polynomial with only pure imaginary zeros) which have only pure imaginary zeros by standard results for polynomials (e.g. [M, pp. 62-63]).

Proof OF NECESSITY. We now assume that $Z_{b}$ has only pure imaginary zeros; it is also even, strictly positive for real $z$, and $O\left(\exp \left(|z|^{2} / 4 b\right)\right)$ for complex $z$ so that it is entire of at most order two and finite type. It follows by standard entire function techniques (see [N2, Proposition 2] for a more complete discussion) that

$$
Z_{b}(z)=\bar{K}_{b} \exp \left(\bar{d}_{b} z^{2}\right) \prod_{j}\left(1+z^{2} / \bar{a}_{j}(b)^{2}\right)
$$

with $\bar{K}_{b}>0, \bar{d}_{b}$ real, $\bar{a}_{j}(b)>0$, and $\Sigma\left(1 / \bar{a}_{j}(b)^{2}\right)<\infty$; here $\pm i \bar{a}_{j}(b)$ are the zeros of $Z_{b}$.

The key idea of the proof is to express $\rho$ as the weak limit of measures $\rho_{b}$ as $b \rightarrow \infty$ (i.e. $\int f(t) d \rho_{b}(t) \rightarrow \int f(t) d \rho(t)$ for all bounded continuous $f$ ) where $\rho_{b}$ is the convolution of $\rho$ with the Gaussian probability measure of mean zero and variance $1 / b$ :

$$
\begin{aligned}
d \rho_{b} / d t & =\int_{-\infty}^{\infty}(b / 2 \pi)^{1 / 2} \exp \left(-b(t-s)^{2} / 2\right) d \rho(s) \\
& =(b / 2 \pi)^{1 / 2} \exp \left(-b t^{2} / 2\right) Z_{b / 2}(b t) \\
& =K_{b} \exp \left(-d_{b} t^{2}\right) \prod_{j}\left(1+t^{2} / a_{j}(b)^{2}\right)
\end{aligned}
$$

with $K_{b}>0, d_{b}>0$ (since $\rho_{b}$ is finite), $a_{j}(b)>0$, and $\Sigma\left(1 / a_{j}(b)\right)^{2}<\infty$. It only remains to show that any finite measure $\rho$, which is the weak limit of measures such as those given by (2.2), must be either as in (1.7) or as in (1.8).

We first make several definitions and then complete the proof after a series of lemmas. We define

$$
A_{b}=\Sigma_{1} 1+\Sigma_{2}\left(1 / a_{j}(b)\right)^{4} ; \quad B_{b}=d_{b}-\Sigma_{2}\left(1 / a_{j}(b)\right)^{2},
$$

where $\Sigma_{1}$ (resp. $\Sigma_{2}$ ) denotes the sum over all $j$ such that $a_{j}(b)<1$ (resp. $\left.a_{j}(b) \geqslant 1\right)$; we further define $f_{b}(t)=d \rho_{b} / d t$ and $V_{b}=-\ln \left(f_{b}\right)$ so that

$$
\begin{gathered}
V_{b}^{\prime}(t)=2 t\left[d_{b}-\sum_{j}\left\{1 /\left(t^{2}+a_{j}(b)^{2}\right)\right\}\right], \\
\left(V_{b}^{\prime} / 2 t\right)^{\prime}=2 t \sum_{j}\left\{1 /\left(t^{2}+a_{j}(b)^{2}\right)^{2}\right\} .
\end{gathered}
$$

Lemma 1. If $A_{b}$ and $\left|B_{b}\right|$ are uniformly bounded as $b \rightarrow \infty$, then $\rho(t)$ is absolutely continuous with density as in (1.8). 
Proof. We define $P_{b}(t)=\Pi_{1}\left(a_{j}(b)^{2}+t^{2}\right)$ and

$$
Q_{b}(t)=\Pi_{2}\left(1+t^{2} / a_{j}(b)^{2}\right) \exp \left(-t^{2} / a_{j}(b)^{2}\right),
$$

where $\Pi_{1}$ and $\Pi_{2}$ are defined analogously to $\Sigma_{1}$ and $\Sigma_{2}$, so that $f_{b}(t)=$ $\tilde{K}_{b} \exp \left(-B_{b} t^{2}\right) P_{b}(t) Q_{b}(t)$. In order to bound $f_{b}(t)$ below for real $t$ and above for complex $t$, we use the following inequalities:

$$
\begin{aligned}
P_{b}(t) & \geqslant \Pi_{1} t^{2} \geqslant t^{2 A_{b}}, \quad t \in[-1,1] \\
\left|P_{b}(z)\right| & \leqslant \Pi_{1}\left(1+|z|^{2}\right) \leqslant\left(1+|z|^{2}\right)^{A_{b}} \leqslant \exp \left(A_{b}|z|^{2}\right), \quad z \in \mathbf{C} \\
Q_{b}(t) & \geqslant \exp \left(-\Sigma_{2} t^{4} / 2 a_{j}(b)^{4}\right) \geqslant \exp \left(-A_{b} t^{4} / 2\right), \quad t \in \mathbf{R} \\
\left|Q_{b}(z)\right| & \leqslant \exp \left(\Sigma_{2}|z|^{4} / 2 a_{j}(b)^{4}\right) \leqslant \exp \left(A_{b}|z|^{4} / 2\right), \quad z \in \mathbf{C}
\end{aligned}
$$

(2.7) and (2.8) are elementary; (2.9) follows by applying (1.10) to (2.6); and (2.10) may be obtained by applying the inequality

$$
\left|\left(1+z^{2}\right) \exp \left(-z^{2}\right)\right| \leqslant \exp \left(|z|^{4} / 2\right), \quad z \in \mathbf{C},
$$

to (2.6). To derive (2.11), we set $u=|z|^{4}$ and $v=\operatorname{Re}\left(z^{2}\right)$ so that (2.11) is equivalent to $(1+u+2 v)^{1 / 2} \exp (-v) \leqslant \exp (u / 2)$, which is itself equivalent to the elementary inequality $(1+w) \leqslant \exp (w)$ with $w=u+\mathrm{w} v$. Combining (2.7) through (2.10), we have

$$
\begin{aligned}
f_{b}(t) & \geqslant \tilde{K}_{b} t^{2 A_{b}} \exp \left(-B_{b} t^{2}-A_{b} t^{4} / 2\right), \quad t \in[-1,1], \\
\left|f_{b}(z)\right| & \leqslant \tilde{K}_{b} \exp \left(\left[\left|B_{b}\right|+A_{b}\right]|z|^{2}+A_{b}|z|^{4} / 2\right), \quad z \in \mathbf{C} .
\end{aligned}
$$

Now, since $f_{b}(t) d t \rightarrow d \rho$, a finite measure, it follows from (2.12) that $\tilde{K}_{b}$ must be bounded above uniformly in $b$; consequently we have from (2.13) that $f_{b}(z)$ is uniformly bounded on compact subsets of $\mathbf{C}$ and thus, by standard analytic function theory, some subsequence of the $f_{b}$ 's converges uniformly on compacts to an entire function $f(z)$ and clearly $d \rho(t)=f(t) d t$. Now by (2.13), $f$ is entire of (at most) exponential order four and finite type, and since each $f_{b}$ has only pure imaginary zeros, so does $f$ by Hurwitz' Theorem; moreover $f$ is even and nonnegative on the real axis (since $\rho \geqslant 0$ ) so we may conclude by standard entire function theory (analogously to the derivation of (2.1)) that $f$ has the form given in (1.8). The conditions on $\alpha$ and $\beta$ are required simply because $\rho$ is a finite measure.

LEMMA 2. For each $b>0$, there is $a T_{b} \geqslant 0$ with $\lim \sup T_{b}<\infty$ such that $V_{b}^{\prime} \leqslant 0$ on $\left[0, T_{b}\right]$ and $V_{b}^{\prime}>0$ on $\left(T_{b}, \infty\right)$. If for some subsequence $b_{j}$ and some $0<t_{1}<t_{2}<\infty, \quad V_{b_{j}}^{\prime} \rightarrow+\infty \quad$ (resp. $\left.-\infty\right)$ uniformly on $\left[t_{1}, t_{2}\right]$, then $\rho\left(\left(t_{1}, \infty\right)\right)=0\left(\operatorname{resp} . \rho\left(\left(-t_{2}, t_{2}\right)\right)=0\right)$.

Proof. The existence of $T_{b}$ follows from the fact that $V_{b}^{\prime}(0)=0$ while $V_{b}^{\prime}(t) / t$ is increasing on $(0, \infty)$ to $2 d_{b}>0$ as $t \rightarrow \infty$. To see that

$$
\lim \sup T_{b}<\infty
$$


we note that $f_{b}(t)$ is nondecreasing on $\left[0, T_{b}\right]$, so that if $T_{b_{k}} \rightarrow+\infty$ it would follow that for any $s_{1}, s_{2}>0$,

$$
\begin{aligned}
\rho\left(\left[s_{1}, s_{1}+s_{2}\right]\right) & \geqslant \limsup _{k \rightarrow \infty} \rho_{b_{k}}\left(\left[s_{1}, s_{1}+s_{2}\right]\right) \\
& \geqslant \liminf _{k \rightarrow \infty} \rho_{b_{k}}\left(\left(-s_{2}, s_{2}\right)\right) / 2 \geqslant \rho\left(\left(-s_{2}, s_{2}\right)\right) / 2
\end{aligned}
$$

which is impossible for a finite measure $\rho$.

We now consider the case when $V_{b_{j}}^{\prime} \rightarrow+\infty$ on $\left[t_{1}, t_{2}\right]$ and define $D_{j}$ as the minimum of $V_{b_{j}}^{\prime}$ on $\left[t_{1}, t_{2}\right]$ which by assumption tends to $+\infty$ as $j \rightarrow \infty$. By elementary calculus, we have for $t, t+\delta \in\left[t_{1}, t_{2}\right]$ and $\delta>0$, that $V_{b_{j}}(t+\delta)$ $\geqslant V_{b_{j}}(t)+D_{j} \delta$ so that $f_{b_{j}}(t+\delta) \leqslant \exp \left(-D_{j} \delta\right) f_{b_{j}}(t)$; after integrating this last inequality, we have that for $0<\delta<\left(t_{2}-t_{1}\right)$,

$$
\rho_{b_{j}}\left(\left[t_{1}+\delta, t_{2}\right]\right) \leqslant \exp \left(-D_{j} \delta\right) \rho_{b_{j}}\left(\left[t_{1}, t_{2}-\delta\right]\right) .
$$

Now clearly $T_{b_{j}} \leqslant t_{1}$ for sufficiently large $j$ so that eventually $f_{b_{j}}$ is nonincreasing on $\left[t_{1}, \infty\right)$ and thus eventually $\rho_{b_{j}}\left(\left[t_{1}+\delta+\tau, t_{2}+\tau\right]\right) \leqslant \rho_{b_{j}}\left(\left[t_{1}+\delta, t_{2}\right]\right)$ for any $\tau>0$; combining this inequality with (2.15) and taking the limit gives $\rho\left(\left(t_{1}+\delta+\tau, t_{2}+\tau\right)\right)=0$ for any $\tau>0$ so that letting $\delta \rightarrow 0$, we have $\rho\left(\left(t_{1}+\tau, t_{2}+\tau\right)\right)=0$ for any $\tau>0$ which clearly implies that $\rho\left(\left(t_{1}, \infty\right)\right)=$ 0 . The case of $V_{b_{j}}^{\prime} \rightarrow-\infty$ is handled essentially identically with the assistance of (2.14) for $s_{1}+s_{2}<t_{2}$.

Lemma 3. If $\lim \sup A_{b}=+\infty$, then there is $a t_{0} \geqslant 0$ and a subsequence $b_{j}$ such that $V_{b_{j}}^{\prime} \rightarrow+\infty$ (resp. $\left.-\infty\right)$ uniformly on compact subsets of $\left(t_{0}, \infty\right)$ (resp. $\left.\left(0, t_{0}\right)\right)$.

Proof. We choose the subsequence $b_{j}$ so that $A_{b_{j}} \rightarrow+\infty$ while $T_{b_{j}}$ has a limit $t_{0} \geqslant 0$; this can be done since lim sup $T_{b}<\infty$ by Lemma 2 . The conclusions then follow by elementary arguments from the inequality (easily derivable from (2.5)) $\left(V_{b}^{\prime} / 2 t\right)^{\prime} \geqslant 2 A_{b} t /\left(1+t^{2}\right)^{2}$, and the elementary calculus fact that

$$
V_{b}^{\prime}\left(t_{2}\right)=\left(t_{2} / t_{1}\right) V_{b}^{\prime}\left(t_{1}\right)+t_{2} \int_{t_{1}}^{t_{2}}\left(V_{b}^{\prime} / s\right)^{\prime} d s .
$$

LEMMA 4. If $A_{b}$ is uniformly bounded as $b \rightarrow \infty$, but $\lim \sup B_{b}=+\infty$ (resp. $\left.\lim \inf B_{b}=-\infty\right)$, then there is a subsequence $b_{j}$ such that $V_{b_{j}}^{\prime} \rightarrow+\infty$ (resp. $-\infty)$ uniformly on compact subsets of $(0, \infty)$.

Proof. We choose $b_{j}$ so that $B_{b_{j}} \rightarrow+\infty$ (resp. $-\infty$ ) and then use the estimate,

$$
\begin{aligned}
-A_{b} / t^{2} & \leqslant-\Sigma_{1}\left(1 /\left(t^{2}+a_{j}(b)^{2}\right)\right) \\
& \leqslant \Sigma_{2}\left(1 / a_{j}(b)^{2}\right)-\sum_{j}\left(1 /\left(t^{2}+a_{j}(b)^{2}\right)\right) \\
& \leqslant \Sigma_{2}\left\{t^{2} /\left(a_{j}(b)^{2}\left(t^{2}+a_{j}(b)^{2}\right)\right)\right\} \leqslant A_{b} t^{2}
\end{aligned}
$$


together with (2.4) to obtain

$$
-A_{b} / t^{2} \leqslant V_{b}^{\prime}(t) / 2 t-B_{b} \leqslant A_{b} t^{2}
$$

which clearly implies the desired result.

Completion of PROOF of TheOREM 2. The theorem is a straightforward consequence of the above four lemmas. We only point out that by combining Lemmas 4 and 2, it follows that we cannot have $A_{b}$ uniformly bounded while lim inf $B_{b}=-\infty$ (or else $\rho$ would be identically zero); on the other hand, when $A_{b}$ is bounded and $\lim \sup B_{b}=+\infty$, then $\rho(t)=K \delta(t)$.

ACKNOWLEDGEMENT. The author has benefited from discussions with many people during the course of this research including, in particular, J. Klauder, J. Lebowitz and D. Ruelle.

\section{REFERENCES}

[B] N. G. de Bruijn, The roots of trigonometric integrals, Duke Math. J. 17 (1950), 197-226. MR $12,250$.

[M] M. Marden, The geometry of the zeros of a polynomial in a complex variable, Math. Surveys, no. 3, Amer. Math. Soc., Providence, R. I., 1949. MR 11, 101.

[N1] C. M. Newman, Zeros of the partition function for generalized Ising systems, Comm. Pure Appl. Math. 27 (1974), 143-159.

[N2] , Inequalities for Ising models and field theories which obey the Lee-Yang theorem, Comm. Math. Phys. 41 (1975), 1-9.

[N3] _ Classifying general Ising models, Les Méthodes Mathématiques de la Théorie Quantique des Champs, C.N.R.S., Paris, 1976, pp. 273-288.

[P] G. Pólya, Collected papers, Vol, II (R. P. Boas, editor), M.I.T. Press, Cambridge, 1974.

[SG] B. Simon and R. B. Griffiths, The $\left(\phi^{4}\right)_{2}$ field theory as a classical Ising model, Comm. Math. Phys. 33 (1973), 145-164.

[T] E. C. Titchmarsh, The theory of the Riemann Zeta-function, Oxford Univ. Press, Oxford, 1951. MR 13, 741 .

Departments of Mathematics and Physics, Technion-Israel Institute of Technology, HAIFA, ISRAEL

Current address: Department of Mathematics, Indiana University, Bloomington, Indiana 47401 